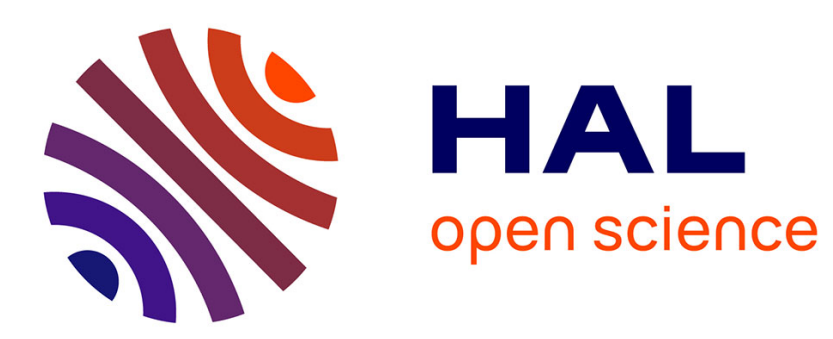

\title{
Mesure de vies moyennes dans le noyau 55Fe par la méthode du parcours de recul
}

J.P. Stoquert, G. Chouraqui, Th. Muller, M. Port, J.M. Thirion

\section{To cite this version:}

J.P. Stoquert, G. Chouraqui, Th. Muller, M. Port, J.M. Thirion. Mesure de vies moyennes dans le noyau 55Fe par la méthode du parcours de recul. Journal de Physique, 1973, 34 (4), pp.295-299. 10.1051/jphys:01973003404029500 . jpa-00207383

\section{HAL Id: jpa-00207383 https://hal.science/jpa-00207383}

Submitted on 1 Jan 1973

HAL is a multi-disciplinary open access archive for the deposit and dissemination of scientific research documents, whether they are published or not. The documents may come from teaching and research institutions in France or abroad, or from public or private research centers.
L'archive ouverte pluridisciplinaire HAL, est destinée au dépôt et à la diffusion de documents scientifiques de niveau recherche, publiés ou non, émanant des établissements d'enseignement et de recherche français ou étrangers, des laboratoires publics ou privés. 


\author{
Classification \\ Physics Abstracts \\ $12.10-12.17-12.37$
}

\title{
MESURE DE VIES MOYENNES DANS LE NOYAU ${ }^{55} \mathrm{Fe}$ PAR LA MÉTHODE DU PARCOURS DE RECUL
}

\author{
J. P. STOQUERT, G. CHOURAQUi, Th. MULlER, \\ M. PORT et J. M. THIRION \\ Laboratoire de Physique du Noyau et de Physique des Particules \\ Centre de Recherches Nucléaires, \\ F-67037 Strasbourg-Cédex
}

(Reçu le 7 septembre 1972, revisé le 6 novembre 1972)

\begin{abstract}
Résumé. - La méthode du parcours de recul a été utilisée pour mesurer les vies moyennes de 3 états excités du noyau ${ }^{55} \mathrm{Fe}$ produit dans la réaction ${ }^{52} \mathrm{Cr}(\alpha, n \gamma)^{55} \mathrm{Fe}$ à $\mathrm{E}_{\alpha}=11 \mathrm{MeV}$. Les résultats (en ps) $-\tau(931 \mathrm{keV})=13,4 \pm 4, \tau(1316)=26 \pm 3,5, \tau(1408)=69 \pm 7,5-$ sont comparés à ceux obtenus par d'autres méthodes ainsi qu'aux prévisions théoriques.

Abstract. - The recoil-distance measurements of mean lives of 3 excited states in ${ }^{55} \mathrm{Fe}$ nucleus produced in the ${ }^{52} \mathrm{Cr}(\alpha, n \gamma){ }^{55} \mathrm{Fe}$ reaction at $\mathrm{E}_{\alpha}=11 \mathrm{MeV}$ are reported. The results (in ps) $-\tau(931 \mathrm{keV})=13.4 \pm 4, \tau(1316)=26 \pm 3.5, \tau(1408)=69 \pm 7.5-$ are compared to those obtained by other methods and to theoretical previsions.
\end{abstract}

1. Introduction. - Le noyau de ${ }^{55} \mathrm{Fe}$ proche de la couche doublement fermée $1 \mathrm{f} 7 / 2$ a fait l'objet de divers calculs dans le cadre du modèle en couches avec un cœur de ${ }^{48} \mathrm{Ca}$ [1], [2], [3], et dans le cadre du modèle à couplage intermédiaire [4], [5], [6], [7], tenant compte d'excitations de neutrons dans les couches 2 p $3 / 2,1 \mathrm{f} 5 / 2,2$ p $1 / 2$. Certains niveaux attribués à des configurations de trous de neutrons couplés à un cœur de ${ }^{56} \mathrm{Fe}$ [8], [9], [10] ne sont pas retrouvés par ces calculs. Par ailleurs, parmi les vies moyennes déterminées expérimentalement [11], [12], [13], certaines valeurs sont en désaccord. Dans le but d'apporter des informations plus précises, nous avons mesuré les vies moyennes des deuxième, troisième et quatrième niveaux excités du ${ }^{55} \mathrm{Fe}$ situés respectivement à $931 \mathrm{keV}, 1316 \mathrm{keV}$ et $1408 \mathrm{keV}$, en utilisant la réaction ${ }^{52} \mathrm{Cr}(\alpha, n \gamma){ }^{55} \mathrm{Fe}$ et la méthode du parcours de recul.

2. Le dispositif expérimental. - Pour un ensemble de noyaux émis de la cible avec la vitesse $v$ à la distance $D$ d'un stoppeur, la méthode du parcours de recul consiste à mesurer

$$
R=\frac{I_{0}}{I_{0}+I_{\mathrm{s}}}=\mathrm{e}^{-D / v \tau}
$$

en fonction de $D . I_{\mathrm{s}}$ et $I_{0}$ sont les intensités des composantes rapide et lente émises respectivement par des noyaux de vitesse $v$ reculant dans le vide et par des noyaux au repos dans le stoppeur ; $\tau$ est la vie moyenne du niveau étudié.
L'application de la méthode nécessite la mise au point d'un dispositif expérimental permettant le réglage et la mesure précise des distances et assurant la planéité et le parallélisme des surfaces de la cible et du stoppeur.

Le schéma du dispositif que nous avons réalisé est représenté sur la figure 1 . A, B et $\mathrm{C}$ sont respectivement le support du stoppeur, le porte-cible et le tendeur de cible et peuvent coulisser sur les tiges $T_{1}$ et $T_{2}$. C est avancé vers $B$ à l'aide du bouton moleté $M_{2}$ pour tendre la cible et les déplacements de $\mathrm{A}$ se font à l'aide du bouton moleté $M_{1}$ situé en dehors de la boîte de cible.

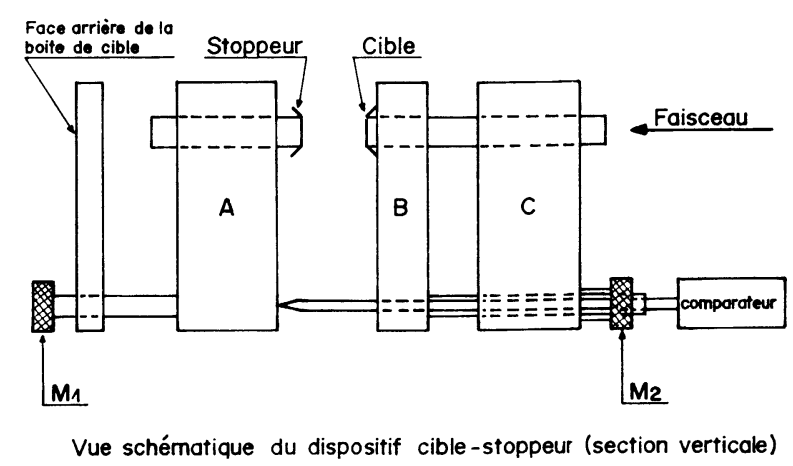

FIG. 1. - Vue schématique du dispositif cible-stoppeur.

Les cibles utilisées sont constituées d'un dépôt d'environ $50 \mu \mathrm{g} / \mathrm{cm}^{2}$ de chrome métallique (composition isotopique naturelle : $83,76 \%$ de ${ }^{52} \mathrm{Cr}$ ) sur un support de mylar de $600 \mu \mathrm{g} / \mathrm{cm}^{2}$. La perte d'énergie 
de particules $\alpha$ de $11 \mathrm{MeV}$ dans ce support est d'environ $300 \mathrm{keV}$. Une pastille de tantale poli sert de stoppeur. Un comparateur solidaire de B et C, permet la mesure des déplacements du stoppeur avec la précision du micron. Le réglage du zéro de ce comparateur était effectué en rapprochant au maximum le stoppeur de la cible. L'écartement était contrôlé en observant sur un écran l'image de diffraction donnée par un Laser He-Ne.

Cette méthode permet difficilement de chiffrer les défauts de planéité et de parallélisme qui entraînent une incertitude sur la valeur absolue de la distance cible-stoppeur dont il sera fait mention plus loin.

3. Exploitation. - La relation (2.1) représente la courbe théorique. En pratique, il faut tenir compte des dispersions des vitesses et des distances de recul, ainsi que de la géométrie finie des compteurs [14]. En considérant la dispersion des vitesses de recul, la relation devient :

$$
R=\frac{I_{0}}{I_{0}+I_{\mathrm{s}}}=\psi(f) \mathrm{e}^{-D / \iota \tau}
$$

où $\psi(f)$ représente le terme de correction. On montre que

$$
\psi(f)=\sum_{k} \alpha_{k} M_{k}(f)
$$

où $M_{k}(f)$ est le moment d'ordre $k$ de la distribution des vitesses et $\alpha_{k}$ un coefficient qui dépend de $D$. Pour que les corrections soient faibles, il faut d'une part que la dispersion des vitesses soit faible $\left(M_{k}(f)\right.$ petit), d'autre part que la distance $D$ soit de l'ordre de grandeur du produit $v \tau$. Avec ces hypothèses, nous posons

$$
\psi(f)=\mathrm{e}^{-D_{\jmath}^{\prime} / v \tau}
$$

où $D_{0}^{\prime}$ est une constante.

De la même manière, la dispersion des distances de recul s'exprimera par

$$
\psi(g)=\mathrm{e}^{-D_{0}^{\prime \prime} / v \tau} .
$$

Finalement, l'ensemble de ces corrections s'effectue en posant

$$
\frac{I_{0}}{I_{0}+I_{\mathrm{s}}}=\mathrm{e}^{-\left(D_{0}+D\right) / v \tau}
$$

où $D_{0}$ groupe les corrections $D_{0}^{\prime}$ et $D_{0}^{\prime \prime}$ et un terme analogue englobant les incertitudes de réglage de zéro du comparateur, ainsi que les défauts de planéité et de parallélisme. Il reste alors sur $D$ l'incertitude d'un micron du comparateur (lecture de la graduation et reproductibilité des déplacements).

La correction due à l'ouverture angulaire du détecteur $\mathrm{Ge}(\mathrm{Li})$ s'exprime par

$$
E_{\mathrm{d}}=E_{0}\left[1+\beta \cos \theta\left(\frac{1+\cos ^{8} \theta_{\mathrm{c}}}{2}\right)\right]
$$

où $E_{0}(1+\beta \cos \theta)$ est l'énergie d'un rayonnement $\gamma$ détecté à l'angle $\theta$ par rapport à la direction de recul et $\theta_{\mathrm{c}}$ le demi-angle au sommet du cône de détection. Dans notre cas $\left(\theta_{c}=15^{\circ}\right)$, cette correction est inférieure à $1 \%$, pour une corrélation isotrope entre le rayonnement $\gamma$ et le noyau de recul.

A partir d'une série de mesures de $I_{0}$ et $I_{\mathrm{s}}$ en fonction de $D$, nous avons déterminé la vie moyenne $\tau$, ainsi que le paramètre $D_{0}$, qui groupe les différentes corrections, par un lissage en moindres carrés de la courbe

$$
\frac{I_{0}}{I_{0}+I_{\mathrm{s}}}=\mathrm{e}^{-\left(D_{0}+D\right) / v \tau} \text {. }
$$

Le programme de lissage détermine également l'écart type sur les valeurs trouvées de $D_{0}$ et $\tau$.

Dans le cas où le niveau étudié de vie moyenne $\tau_{1}$ est alimenté à la fois directement et par l'intermédiaire d'une cascade provenant d'un niveau supérieur de vie moyenne $\tau_{2}$, il faut retrancher aux composantes $I_{0}$ et $I_{\mathrm{s}}$ observées les contributions dues à l'alimentation par le niveau supérieur, à l'aide des relations suivantes :

$$
\begin{aligned}
I & =I_{0}+I_{\mathrm{s}} \\
I_{\mathrm{s}}\left(\tau_{1}\right) & =I_{\mathrm{s}}-\alpha \cdot I . P_{D} \\
I_{0}\left(\tau_{1}\right) & =I_{0}-\alpha \cdot I .\left(1-P_{D}\right) \\
P_{D} & =1-\mathrm{e}^{-D / v_{2} \tau_{2}}-\left[1-\mathrm{e}^{-\left(1-v_{2} \tau_{2} / v_{1} \tau_{1}\right) \cdot D / v_{2} \tau_{2}}\right] \times \\
& \times \mathrm{e}^{-D / v_{1} \tau_{1}} /\left(1-v_{2} \tau_{2} / v_{1} \tau_{1}\right)
\end{aligned}
$$

où $P_{D}$ est la probabilité du niveau étudié d'émettre un rayonnement $\gamma$ dans la composante $I_{\mathrm{s}}$ et provenant de la désexcitation du niveau supérieur par cascade $\gamma$, le stoppeur étant placé à la distance $D . I_{0}\left(\tau_{1}\right)$ et $I_{\mathrm{s}}\left(\tau_{1}\right)$ sont les composantes corrigées dues uniquement à l'alimentation directe, et $\alpha$ est la fraction de $I$ provenant du niveau supérieur.

Le coefficient $\alpha$ inclut le rapport d'alimentation par neutrons des niveaux 1 et 2 et la corrélation angulaire $\gamma-\gamma$ de la cascade. Les valeurs les plus probables de ce paramètre et de la vie moyenne $\tau_{1}$, connaissant $\tau_{2}$, sont déterminées par un programme d'itération.

De plus, par cette méthode d'exploitation, il est également possible de tenir compte d'une éventuelle contamination du stoppeur par la cible. En effet, l'alimentation d'un niveau par un autre de vie moyenne nettement supérieure simule la contamination, les rayonnements $\gamma$ étant dans les deux cas émis par des noyaux au repos dans le stoppeur. Le coefficient $\alpha$ inclut alors le facteur de contamination.

Les valeurs des composantes $I_{0}$ et $I_{\mathrm{s}}$ sont extraites de chaque spectre par lissage d'une portion du spectre avec deux gaussiennes et un fond linéaire. Le choix de ce type de lissage pour la composante $I_{\mathrm{s}}$, qui est un pic dissymétrique, est cependant justifié en raison de la résolution instrumentale qui est de l'ordre de $3 \mathrm{keV}$ tandis que la séparation de $I_{0}$ et $I_{\mathrm{s}}$ est de 4,5 à $6 \mathrm{keV}$ selon la transition étudiée. L'erreur sur les 
valeurs $I_{0}$ et $I_{\mathrm{s}}$ déduites du lissage est calculée en tenant compte de l'erreur statistique sur les taux de comptage et de la qualité de l'ajustement obtenu. Deux exemples de décomposition de spectre sont donnés figure 2.
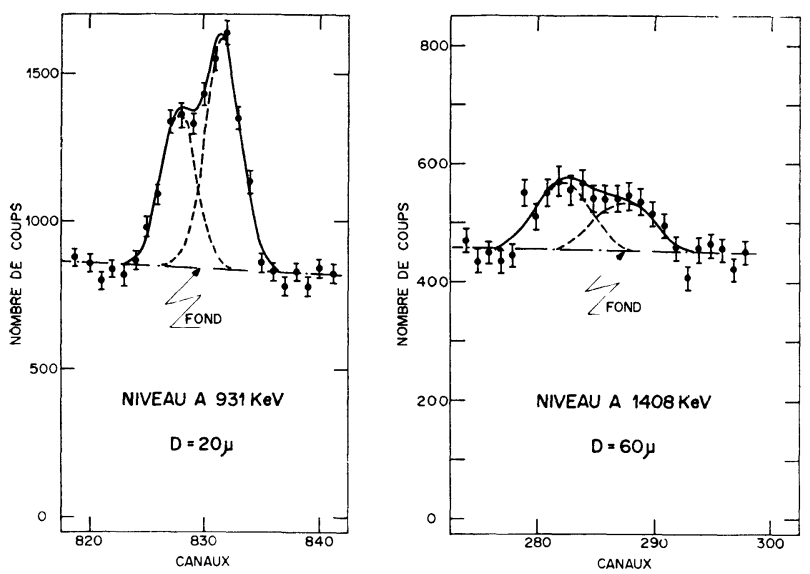

FIG. 2. - Exemples de décomposition de spectres permettant de déterminer les valeurs de $I_{0}$ et $I_{\mathrm{S}}$ (deux gaussiennes et un fond linéaire).

4. Résultats expérimentaux. - Ce travail a été réalisé à l'aide du faisceau ${ }^{4} \mathrm{He}^{++}$délivré par l'accélérateur Van de Graaff de 5,5 MeV du Groupe de Laboratoires de Strasbourg-Cronenbourg. L'intensité du faisceau était limitée à $0,02 \mu \mathrm{A}$ pour éviter la détérioration du support de cible en mylar.

Les rayonnements $\gamma$ étaient détectés à $0^{\circ}$ dans un compteur au $\mathrm{Ge}(\mathrm{Li})$ de $54 \mathrm{~cm}^{3}$ et de $3 \mathrm{keV}$ de résolution pour les raies standard du ${ }^{60} \mathrm{Co}$. Dans les spectres directs relevés à l'énergie incidente de $11 \mathrm{MeV}$, correspondant à un minimum du bruit de fond dû à la réaction ${ }^{12} \mathrm{C}\left(\alpha, \alpha^{\prime} \gamma\right){ }^{12} \mathrm{C}$ (rayonnement $\gamma$ de $4,43 \mathrm{MeV}$ ) [15], les seules raies $\gamma$ provenant du ${ }^{55} \mathrm{Fe}$ correspondaient aux transitions entre les 4 premiers niveaux excités (voir Fig. 3 et [16] à [20]). Nous avons vérifié l'attribution de ces raies à la réaction ${ }^{52} \mathrm{Cr}(\alpha, n \gamma){ }^{55} \mathrm{Fe}$ $(Q=-3,58 \mathrm{MeV})$ à l'aide d'un spectre de coïncidences $n-\gamma$. Les neutrons étaient détectés par un grand scintillateur liquide $\mathrm{Ne} 213 \mathrm{de} 25 \mathrm{~cm}$ de diamètre et $4 \mathrm{~cm}$ d'épaisseur. Cependant le rendement de la réaction ne permettait pas d'effectuer une série de spectres en coïncidence avec un taux de comptage suffisant. Les composantes $I_{0}$ et $I_{\mathrm{s}}$ des raies $\gamma$ correspondant aux transitions étudiées ont donc été extraites des spectres directs relevés pour différentes valeurs de la distance $D$.

Dans ces conditions le demi-angle au sommet du cône de recul des noyaux ${ }^{55} \mathrm{Fe}$ est de $20^{\circ}$. La vitesse moyenne calculée pour une distribution isotrope des neutrons est $v / c=0,55 \%$. La vitesse moyenne expérimentale, déduite du déplacement Doppler observé, est de 0,48 ou $0,49 \%$ selon les transitions. La différence entre la valeur calculée et ces mesures est due à l'influence de la corrélation angulaire $n-\gamma$ compte tenu de sa perturbation par recul dans le vide. Pour

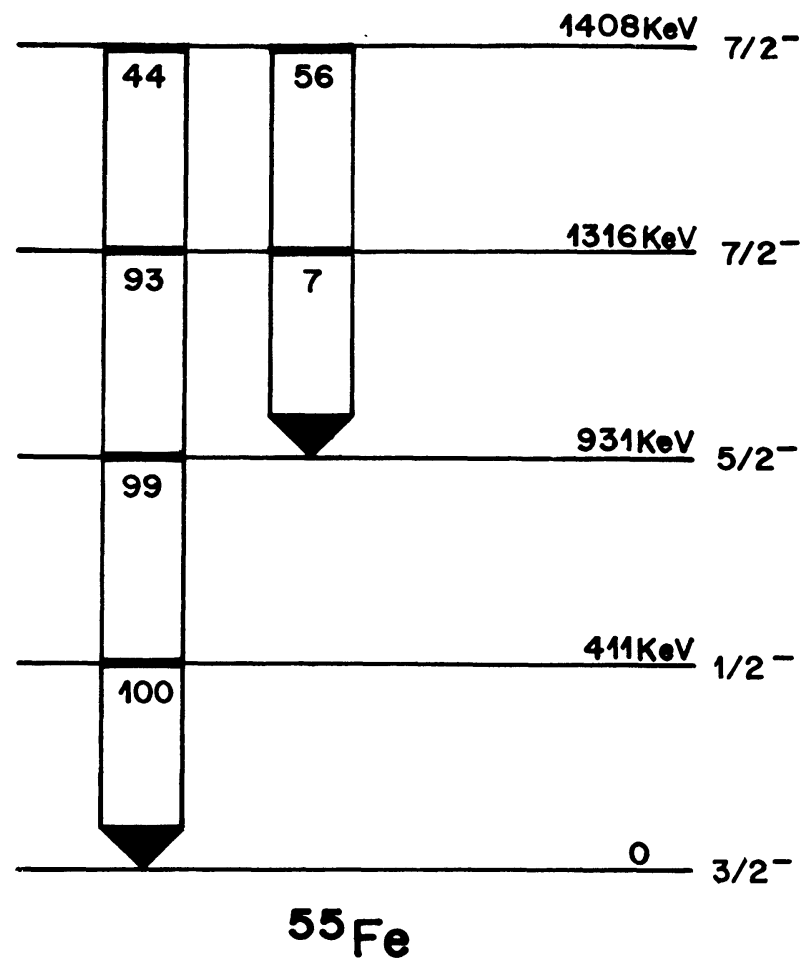

FIG. 3. - Schéma de désexcitation des premiers niveaux du noyau ${ }^{55} \mathrm{Fe}$. Les intensités relatives sont celles reportées dans Nuclear Data Sheets [18].

tenir compte de cette incertitude, nous avons utilisé pour le calcul de $\tau$ la valeur $v / c=0,5 \%$ avec une erreur relative de $10 \%$.

Les résultats obtenus sont illustrés pour chaque niveau étudié par une série de mesures (Fig. 4, 5 et 6). Les valeurs reportées dans le tableau I sont des moyennes sur deux séries de mesures. Le coefficient $\alpha$ de 0,40 pour le niveau à $931 \mathrm{keV}$ est obtenu en introduisant dans le calcul l'alimentation de ce niveau par la cascade $\gamma$ à partir du niveau à $1408 \mathrm{keV}$, en prenant pour vie moyenne de ce dernier, la valeur mesurée de 69 ps. Les valeurs $\alpha=0$ pour les niveaux à 1316 et $1408 \mathrm{keV}$ sont obtenues en supposant une alimentation par l'intermédiaire d'un niveau de vie très longue

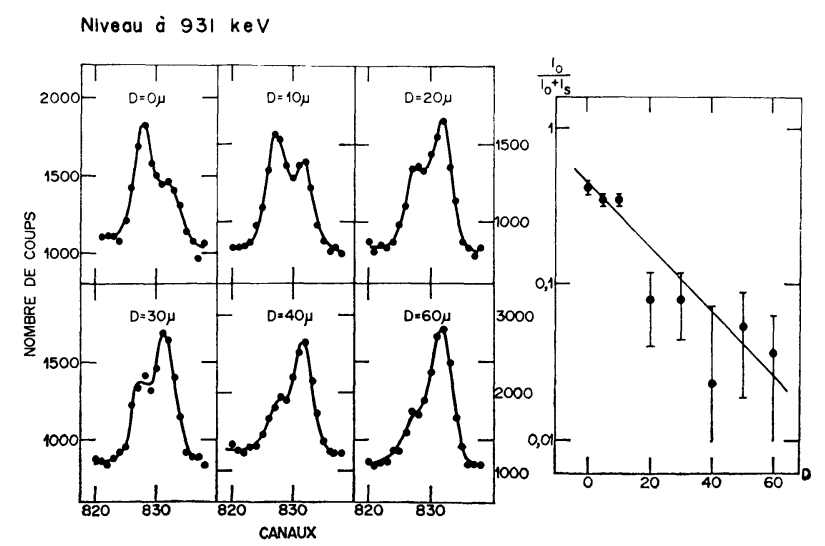

FIG. 4. - Spectres donnant $I_{0}$ et $I_{\mathrm{S}}$ et évolution du rapport $I_{0} /\left(I_{0}+I_{\mathrm{s}}\right)$ en fonction de la distance $D$ pour le niveau à $931 \mathrm{keV}\left(E_{\gamma}=931 \mathrm{keV}\right)$. 
Niveau ì $1316 \mathrm{keV}$
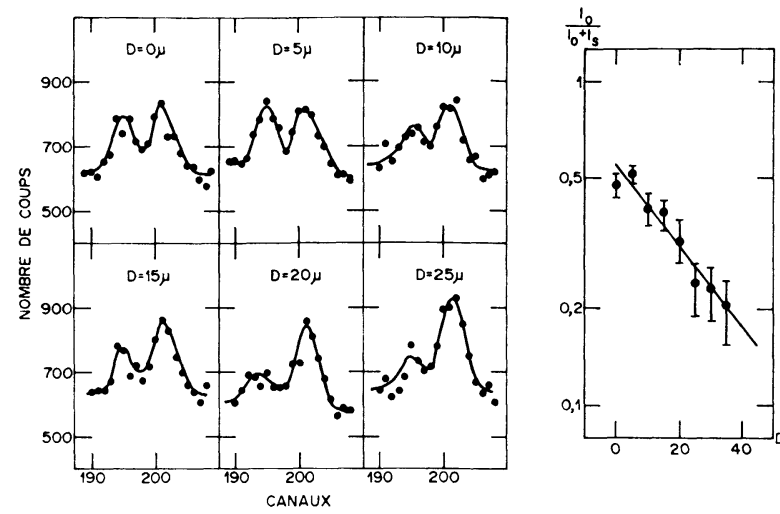

FIG. 5. - Spectres donnant $I_{0}$ et $I_{\mathrm{S}}$ et évolution du rapport $I_{0} /\left(I_{0}+I_{\mathrm{S}}\right)$ en fonction de la distance $D$ pour le niveau à $1316 \mathrm{keV}\left(E_{\gamma}=1316 \mathrm{keV}\right)$.

\begin{tabular}{|c|c|c|c|c|}
\hline $\begin{array}{c}\text { Energie } \\
\text { du niveau } \\
\mathrm{keV}\end{array}$ & $v / c$ & $\begin{array}{c}D_{0} \\
\mu\end{array}$ & $\alpha$ & $\begin{array}{c}\tau \\
\text { ps }\end{array}$ \\
\hline - & - & $\underline{-}$ & - & - \\
\hline 931 & 0,48 & $17 \pm 7$ & 0,40 & $13,4 \pm 4,0$ \\
\hline 1316 & 0,48 & $30 \pm 7$ & 0 & $26 \pm 3,5$ \\
\hline 1408 & 0,49 & $-2 \pm 6$ & 0 & $\pm 7,5$ \\
\hline
\end{tabular}

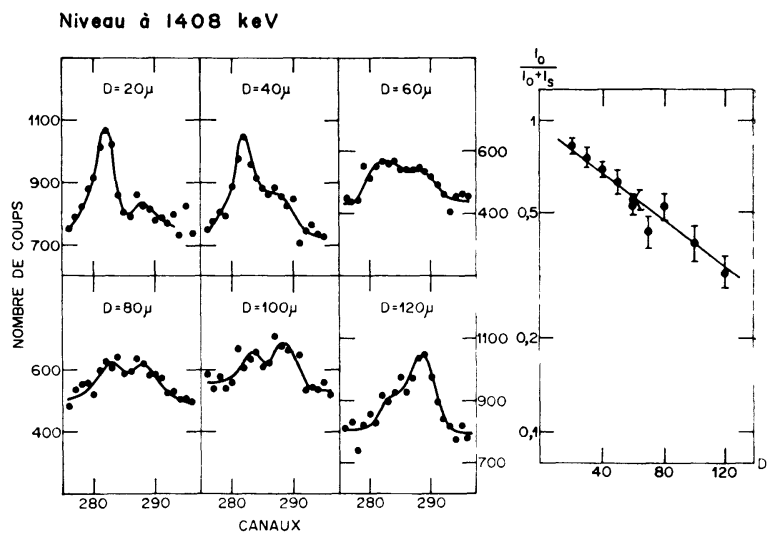

FIG. 6. - Spectres donnant $I_{0}$ et $I_{\mathrm{S}}$ et évolution du rapport $I_{0} /\left(I_{0}+I_{\mathrm{s}}\right)$ en fonction de la distance $D$ pour le niveau à $1408 \mathrm{keV}\left(E_{\gamma}=1408 \mathrm{keV}\right)$.

pour simuler la contamination du stoppeur par la cible. Les valeurs obtenues montrent que cet effet, s'il existe, est négligeable.

5. Discussion. - L'ensemble des résultats expérimentaux et théoriques est résumé dans le tableau II.

Pendant la réalisation de ce travail d'autres résultats expérimentaux ont été obtenus par des méthodes

\section{TABLEAU II}

Comparaisons des vies moyennes mesurées et calculées (en ps)

Expérimental

\begin{tabular}{|c|c|c|c|c|c|c|}
\hline \multirow[b]{2}{*}{$\begin{array}{c}\text { Energie } \\
\text { du niveau } \\
\text { keV }\end{array}$} & \multicolumn{4}{|c|}{ Expérimental } & \multicolumn{2}{|c|}{ Théorique } \\
\hline & $\begin{array}{l}\text { Atténuation de } \\
\text { dans les solides } \\
\left({ }^{(a)}\right)\end{array}$ & $\begin{array}{l}\text { effet Doppler } \\
\text { dans les gaz } \\
\left({ }^{b}\right)\end{array}$ & $\begin{array}{c}\text { Elec- } \\
\text { tronique } \\
{ }_{(c)}\left({ }^{c}\right)\end{array}$ & $\begin{array}{c}\text { Nos } \\
\text { mesures }\end{array}$ & $\begin{array}{c}\text { Modèle } \\
\text { en couches } \\
\left({ }^{d}\right)\end{array}$ & $\begin{array}{c}\text { Modèle } \\
\text { unifié } \\
\left({ }^{e}\right)\end{array}$ \\
\hline$\overline{0}$ & 一 & - & - & - & $\overline{60}$ & -1 \\
\hline 931 & & $11 \pm 4$ & & $13,4 \pm 4,0$ & 160 & 3,8 \\
\hline 1316 & $0,91 \begin{array}{l}+0,91 \\
-0,31\end{array}$ & $14 \pm 3$ & $<10$ & $26 \pm 3,5$ & 1,4 & 0,56 \\
\hline 1408 & & $\begin{array}{l}40+40 \\
-20\end{array}$ & $49 \pm 10$ & $69 \pm 7,5$ & & \\
\hline
\end{tabular}

$\left({ }^{a}\right)$ Réf. [11]; $\left({ }^{b}\right)$ Réf. [12] ; $\left({ }^{c}\right)$ Réf. [13] ; $\left({ }^{d}\right)$ Réf. [3] ; $\left({ }^{e}\right)$ Réf. [4].

\section{TABLEAU III}

Intensité des transitions en unités de Weisskopf

$\begin{array}{ccc}\begin{array}{c}\text { Energie } \\ \text { du niveau (keV) }\end{array} & |\mathrm{M}(\mathrm{M} 1)|^{2} & |\mathrm{M}(\mathrm{E} 2)|^{2} \\ - & -\overline{-} & - \\ 931 & 2,7.10^{-3} & 0,80 \\ 1316 & & 6,61 \\ 1408 & & 0,08 \\ \left({ }^{a}\right) & 78.10^{-3} & 1,5\end{array}$

Théorique

Expérimental

$\begin{array}{cc}\mid \mathrm{M}(\mathrm{M} 1) & \text { Théorique } \\ - & |\mathrm{M}(\mathrm{E} 2)|^{2} \\ 6.10^{-3} & - \\ & 5,5 \\ & 10,4\end{array}$

$\left({ }^{a}\right)$ Valeurs moyennes des intensités de transition de la compilation de Skorka et al. [22] dans des noyaux $20 \leqslant \mathrm{~A} \leqslant 40$.

Les résultats théoriques sont ceux de Carola et Ohnuma [4]. 
différentes. Pour le niveau à $931 \mathrm{keV}$, la vie moyenne $\tau=13,4 \pm 4,0$ ps que nous avons déterminée confirme la valeur trouvée par Donahue et al. [12] par la méthode d'atténuation dans les gaz. Pour le niveau à $1316 \mathrm{keV}$, le désaccord avec la valeur mesurée par Robertson et al. [11], [13] par la méthode d'atténuation dans les solides est important. Nous avons vérifié que cette méthode ne donne pas de résultats concluants par la réaction ${ }^{52} \mathrm{Cr}(\alpha, n \gamma){ }^{55} \mathrm{Fe}$ que nous avons utilisée ; par ailleurs, une vie moyenne, $\tau=0,91 \mathrm{ps,} \mathrm{est} \mathrm{incom-}$ patible avec les spectres que nous avons observés car $\frac{I_{0}}{I_{0}+I_{\mathrm{s}}}$ serait nul à toutes les distances $D$ pour lesquelles nous avons fait des mesures.

En ce qui concerne le niveau à $1408 \mathrm{keV}$, la vie moyenne $\tau=69 \pm 7,5$ ps est supérieure à celle mesurée par Robertson et al. [13] par la méthode électronique, mais la méthode du parcours de recul semble mieux adaptée aux déterminations de vies moyennes de cet ordre de grandeur.

Le tableau III représente les intensités des transitions en unités de Weisskopf. Pour le niveau à $931 \mathrm{keV}$, la valeur $|\delta|=0,36 \pm 0,11$ [21] a été utilisée.

La comparaison des vies moyennes avec les prévisions théoriques fait essentiellement apparaître la nécessité de calculs tenant compte de plus de configurations, en particulier pour retrouver le niveau à $1408 \mathrm{keV}$, considéré comme un trou de neutrons dans $1 \mathrm{f} 7 / 2$, qui ne rentre pas dans les calculs effectués jusqu'à présent. L'accord avec les résultats du modèle unifié semble meilleur, mais il ne paraît pas possible d'en tirer des conclusions car les paramètres collectifs de ce modèle peuvent suppléer en partie au manque de configurations prises dans le modèle en couches, et des calculs plus complets s'avèrent nécessaires pour trouver un meilleur accord avec l'expérience.

\section{Bibliographie}

[1] Maxwell, J. R., Parkinson, W. C., Phys. Rev. 135 (1964) B 82.

[2] Vervier, J., Nucl. Phys. 78 (1966) 497.

[3] Ohnuma, H., Nucl. Phys. 88 (1966) 273.

[4] Carola, T. P. G., Ohnuma, H., Nucl. Phys. A 165 (1971) 259.

[5] Ramavataram, K., Phys. Rev. 132 (1963) 2255.

[6] Larner, D., Phys. Rev. C 2 (1970) 522.

[7] Csürös, M., Cameron, J. A., Zamori, Z., Can. J. Phys. 49 (1971) 1832.

[8] Goodman, C. D., Ball, J. B., Fulmer, C. B., Phys. Rev. 127 (1962) 574.

[9] Sheer, R., Bayman, B. F., Rost, E., Rickey, M. E., Hoot, C. G., Phys. Rev. 139 (1965) B 1272.

[10] Glashausser, C., Rickey, M. E., Phys. Rev. 154 (1967) 1033.

[11] Robertson, B. C., Carola, T. P. G., Sheppard, D. M., Olsen, W. C., Nucl. Phys. A 160 (1971) 137.

[12] Donahue, D. J., Hersberger, R. L., Phys. Rev. C 4 (1971) 1697.
[13] Robertson, B. C., Neilson, G. C., MacDonald, W. J., Nucl. Phys. A 189 (1972) 439.

[14] Jones, K. W., SchWarzschild, A. Z., Warburton, E. K., Fossan, D. B., Phys. Rev. 178 No 4 (1969) 1773.

[15] Mitchell G. E., Carter, E. B., Davis, R. H., Phys. Rev. 133 (1964) B 1434.

[16] Fischbeck, H. J., Porter, F. T., Freedman, M. S., Wagner, Jr., F., Bolotin, H. H., Phys. Rev. 150 (1966) 941.

[17] Haupt, W., Lange, D., Eckert, H. G., FlammersFELD, A., Z. Phys. 188 (1965) 256.

[18] Nuclear data sheets, National Academy of Sciences, National Research Council, Washington D. C. Nuclear Data B 3 (1970) 3, 4-1 ; 5, 6-37.

[19] Pilt, A. A., Sheppard, D. M., Olsen, W. C., Carola, T. P. G., Twin, P. J., Nucl. Phys. A 150 (1970) 439.

[20] Tepel, J. W., Malan, J. G., de Villiers, J. A. M., Nucl. Phys. A 158 (1970) 129.

[21] Bauer, R. W., Deutsch, M., Nucl. Phys. 16 (1960) 264. 